\title{
Research on Quality Control of Digital Production Lines in Aviation Enterprises
}

\author{
Ce Wang ${ }^{1, a}$, Guijiang Duan ${ }^{1}$, Rui Liu ${ }^{1}$ and Wu Sun ${ }^{2}$ \\ ${ }^{1}$ School of Mechanical Engineering and Automation, Beihang University, Beijing, 100191, China \\ ${ }^{2}$ AVIC Xi'an Aircraft Industrial (Group) Co. Ltd, Xi'an, 710089, China
}

\begin{abstract}
Focusing on the requirements of efficient quality control of digital production lines in aviation enterprises, the framework quality control system of digital production lines were studied in this paper. First, the quality date categories of digital production lines were sorted out, a multi-source quality data acquisition scheme was proposed,. Second, the importance of key quality characteristics in quality control was discussed as well as a solution to find key quality features was established. Again, a multi-channel statistical quality control framework was constructed and a detailed implementation process was proposed. Finally, a digital production line quality control prototype system was designed and developed and validated in an aviation manufacturing enterprise.
\end{abstract}

\section{Introduction}

The continuous development of digital technology such as intelligent sensing, ubiquitous sensing, information integration etc. has made it possible to acquire data in deeper and broader areas, providing technology foundation for the technological transformation of aviation manufacturing enterprises. With the increase in long-life, short cycle time and high-strength performance requirements of new-generation aircraft, the fuselage structure of new-generation aircraft has become increasingly complex, compact, and large-scale. The company's production requirements are difficultly meted by traditional manufacturing technology and production lines. With the "Industry 4.0" and "Made in China 2025 strategy" proposed and advanced, the impact of intelligent manufacturing technology on the manufacturing sector has become increasingly in-depth. Many enterprises have tried to establish digital manufacturing production lines with exemplary functions.

Quality data is an important resource in the quality control system of digital production lines. ${ }^{[1]}$ The establishment of technology system of complete quality data acquisition, analysis, management, decision support and process improvement based on quality data can provide scientific, comprehensive and systematic data support for quality control and quality improvement of digital production lines. It is a great significance for the effective implementation of quality management and risk management and control and an important basic work in the construction of digital manufacturing production lines.

Domestic aviation companies have already carried out some related work on the acquisition, management and analysis of quality data, but there are still some deficiencies, which are manifested in: (1) Data acquisition.
Many companies already have a large number of digital inspection equipment and hand-held digital display equipment with data transmission functions. However, they cannot achieve timely and accurate quality data collection due to the lack of data acquisition software systems; (2) Lack of effective digital methods for data assurance. It cannot make scientific warnings based on quality data due to the lack of analysis and processing about quality data. For some key quality features that require statistical process analysis, only MINITAB or EXCEL software can be used for manual analysis, which is inefficient and cannot fully play the role of product quality data; (3) The use of data is not comprehensive enough. Only the finished inspection quality data can be simply analyzed, however, the on-machine measurement data and process equipment parameters cannot be managed and analyzed.

This paper aims at the characteristics and status quo of the digital production lines of aviation enterprises, builds a multi-source quality data acquisition system based on the existing digitalization, and extracts key quality features, makes full use of multi-source quality data, builds a multi-channel statistical quality control platform for digital production lines, realize the warning of the production status of the digital production line and guarantee the product quality of the digital production line.

\section{Digital production line quality control framework and technical scheme}

The traditional quality control tends to collect, manage, and analyze the data of the finished inspection product, ignoring the quality data generated in the production process. ${ }^{[2]}$ In the manufacturing process of the product, the production equipment continuously generates data 
related to processing such as spindle speed, spindle current, feed speed, etc. These data are directly applied to the product through the production equipment, are the quality data of the digital production line. The intermediate state data measured in the on-machine measurement system during product manufacturing and manufacturing process reflects the state of the product during the processing. The result is the accumulation of the process. These data are the quality data of the production line. In addition, the key processing process parameter data of the processing equipment and onmachine measurement data are larger than the finished

inspection data, and are more close to the production and processing process, and are more able to reflect the production status. The quality control of the digital production line need to collect, manage, and analyze quality data of key process parameters of the processing equipment and on-machine measured as well as the finished inspection quality data, so as to realize the quality control and state warning of the digital production line.

\subsection{Overall framework for quality control of digital production lines}

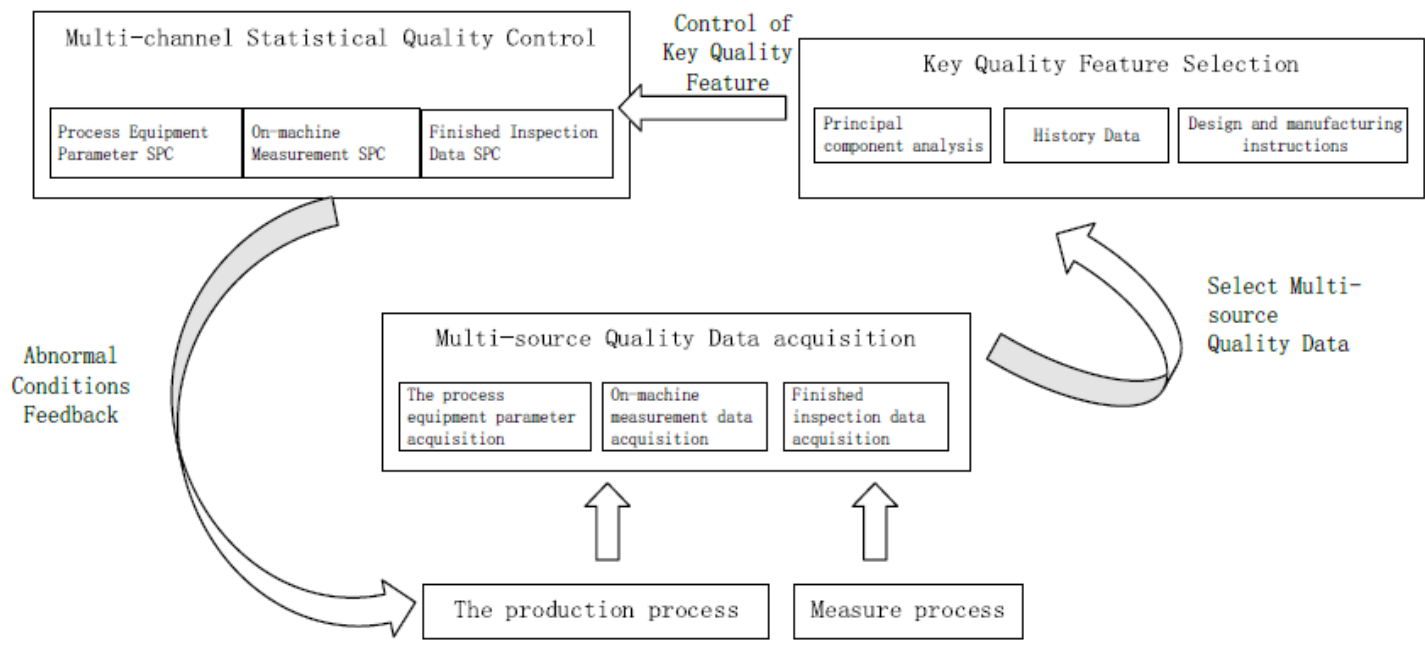

Figure 1. Overall framework for quality control of digital production line

To address the two issues of insufficient data collection capacity and lack of quality control in the quality control of digital production lines, the quality control system built is divided into three modules as shown in Figure 1: (1) multi-source quality data acquisition module: collect multi-source quality data including process parameter data, on-machine measurement data, and finished inspection data;(2) the key quality feature selection module: select the key parameters in the process parameter, the key process in the on-machine measurement, and the key features in the finished inspection; (3)multi-channel statistical quality control module: multi-channel statistical quality control of equipment process parameter data, on-machine measurement data and finished inspection data.

\subsection{Digital Production Line Quality Control Technical Scheme}




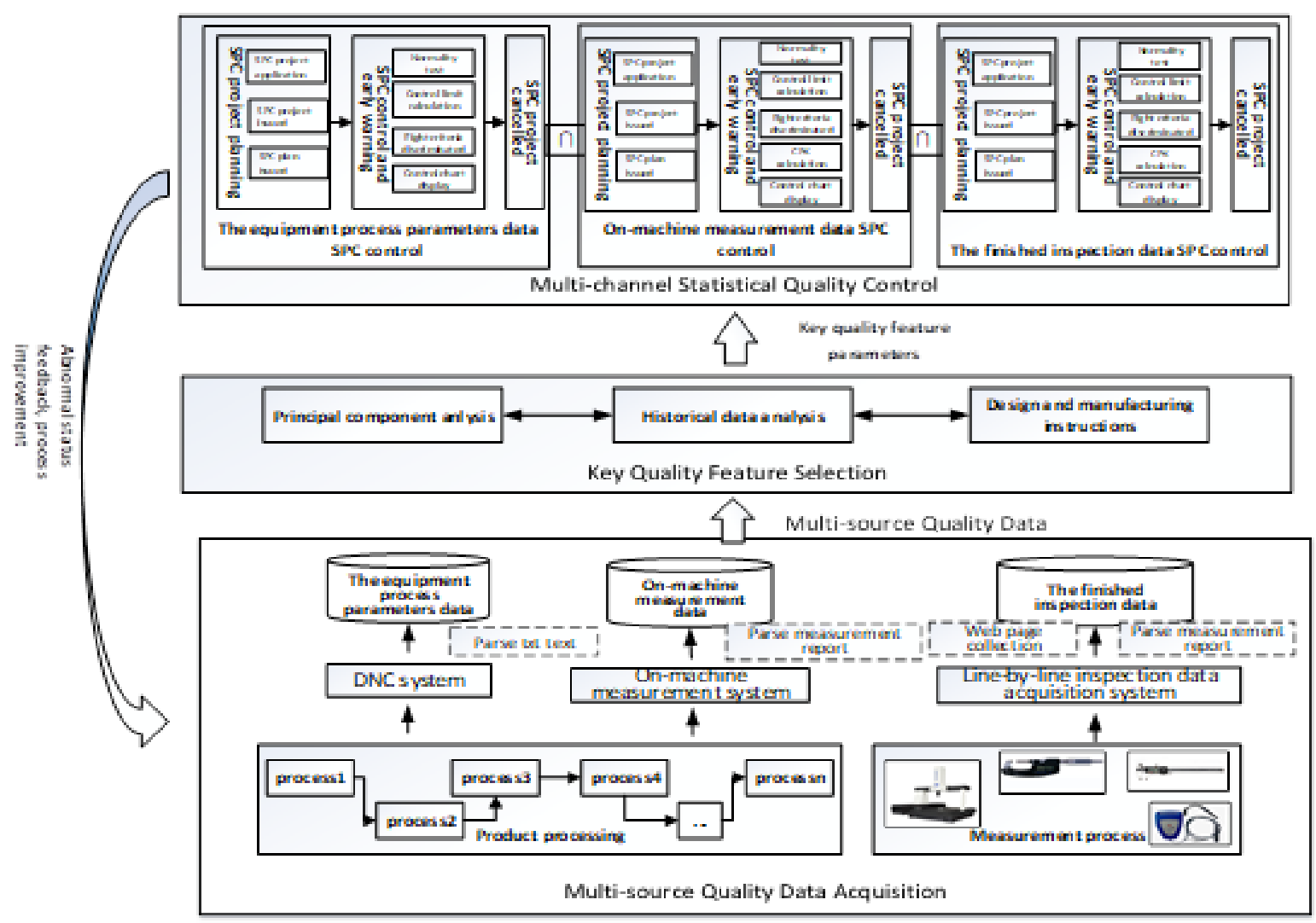

Figure 1. Digital production line quality control technical scheme

To achieve full-scale quality control of the digital production line, it is necessary to control the data generated during product processing and the quality data generated during the measurement process. Based on these requirements for quality control of digital production lines, the technical line for quality control of digital production lines as shown in Figure 2 was established.

Using enterprise DNC system, on-machine measurement system and some existing digital measurement equipment, combined with the data acquisition system built to build a multi-source quality data acquisition system, to realize the acquisition of the processing process parameters, on-machine measurement data during product processing and the acquisition of finished inspection data during the measurement process, and store it in a structured database. ${ }^{[3]}$

The establishment of key quality features depends on a combination of mathematical methods such as principal component analysis, grey correlation analysis, mathematical statistics and historical data, as well as design and manufacturing instructions. Through the above methods, the key parameters, key processes and key features that really play a decisive role in product quality are screened out. ${ }^{[4]}$

Using Statistical Process Control(SPC) as a quality control measure to realize multi-channel statistical quality control of equipment process parameter data, on-machine measurement data, and finished inspection data through SPC control charts and control chart discriminants. ${ }^{[5]}$ Abnormal conditions will be early warned and feedback will be thrown to the product processing process.

\section{Multi-channel statistical process control mode establishment}

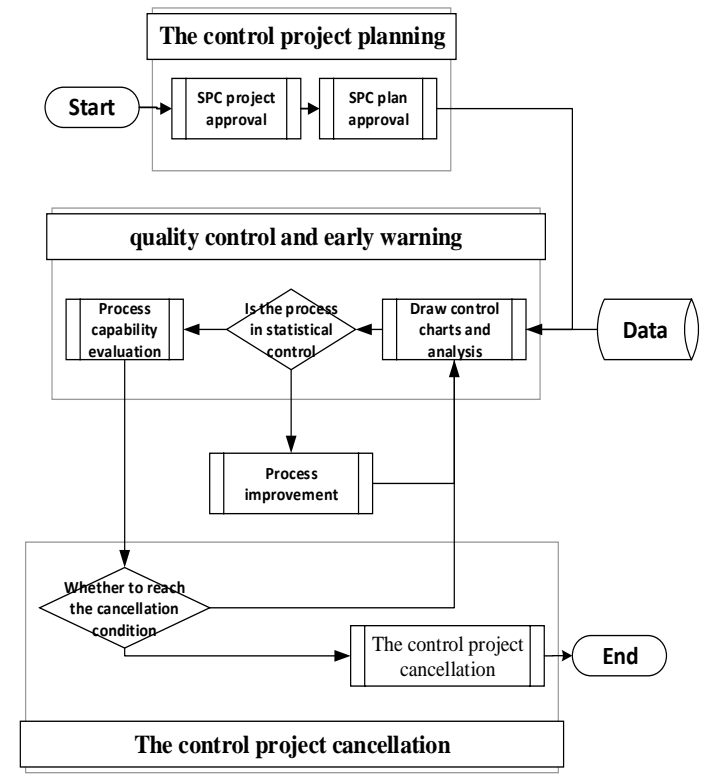

Figure 2. SPC implementation process

SPC is a mature and effective quality control tool that can distinguish between abnormal fluctuations and normal fluctuations in the process and determine whether the process is in a controlled state. ${ }^{[6]}$ The establishment of the three dynamic combination channels including the equipment process parameters SPC control, on-machine 
measurement data SPC control, the finished inspection data SPC control to achieve equipment, process, product trinity of the overall management and control requires to follow the process as shown in Figure 3: the control project planning, quality control and early warning, and the control project cancellation.

There is still a certain distance from the establishment of key quality features and the need to use SPC control. For some features, even if SPC is implemented, the quality of the product cannot be improved; or some features have been in a stable production state for a long time under the existing process technology, and it is no longer necessary to implement SPC. Therefore, before the implementation of SPC, the company needs to do the control project planning.

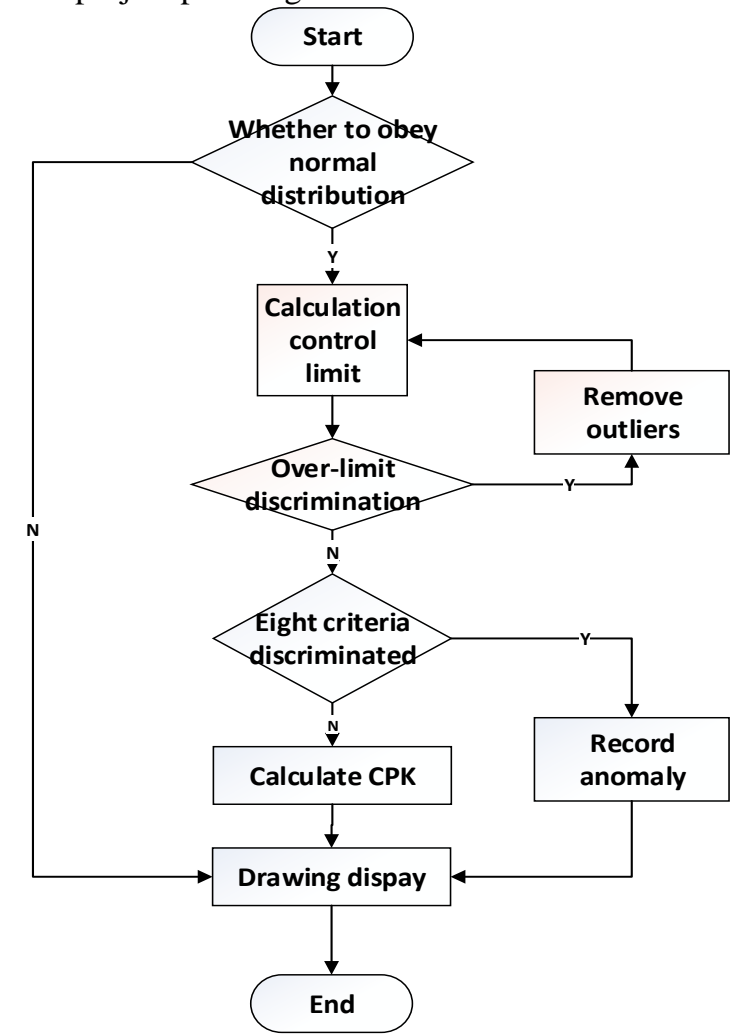

Figure 4. The quality control and early warning process

As shown in Figure 4, the quality control and early warning firstly needs to carry out the normal distribution hypothesis testing, because the calculation of control limits, discriminatory judgments of various discriminatory criteria, and CPK calculation are based on the fact that the data obeys the normal distribution, and do not obey the normal distribution, control limit, early warning, CPK will be meaningless. If the data obey the normal distribution, use the data to calculate the control limits, and judge whether the data exceeds the limit, and if it exceeds, removed it, calculation the control limits again, the control limit will be determined. After that, eight criterion discrepancies are taken and the abnormality is recorded. If there is no abnormality, CPK is calculated and the drawing is finally displayed.$^{[7]}$

As quality control progresses, problems are discovered and resolved, processes are improved, some projects reach a controlled state and are in a controlled state for a long time, such as "CPK is higher than 1.33 for 24 consecutive months" and other conditions are met, at this time, it is no longer significant to continue to carry out quality control of the project, and the cancellation of the control project needs to be considered. In addition, some control projects have long been unable to achieve a controlled level due to their own characteristics or current technological level. For such projects, it is of little significance to continue to control them. It is also necessary to consider the cancellation of such control projects.

\section{Conclusion}

Quality control is an important part of the digital production line. It is of great significance to improve the quality of the company's products. Based on the actual business requirements of the quality control of the digital production lines for aviation companies, this paper builds a quality control framework including three modules: multi-source quality data acquisition module, the key quality feature selection module, multi-channel statistical quality control module. Through the application of digital production line quality control system, the company has improved the company's quality control capabilities, improved the quality of its products, and provided strong support for the company's quality business.

\section{Acknowledgement}

This work was supported by the Graduate Student Innovation Fund of Beihang University (YCSJ-03-201709) and AVIC Xi'an Aircraft Industrial (Group) Co. Ltd. The authors would like express their appreciation to the agency.

\section{References}

1. Yihai.H, Xiaoqing. T, Meiqing.W Computer Integrated Manufacturing Systems 12 1161(2006)

2. Zhonghua Y, Zhaotong W, Chinese Journal of Mechnical Engineering 8 60(2001)

3. A Krenzel. Computer-aided data collection system for assisting in analyzing critical situations. US Patent 5,124,915, (1992)

4. Yihai $\mathrm{H}$, Xiaoqing $\mathrm{T}$ Acta Aeronautica $\mathrm{Et}$ Astronautica Sinica 281468 (2007)

5. Wei $C$, Rui $M$, Zhenge $Y$ Industry Engineering And Management 1 43(2005)

6. Yanqiu L, Junli S, Xueheng T Development \& Innovation of Machinery \& Electric Products 1 69(2008)

7. Xiyuan Q, Jianfen J, Xuxian H Computer Engineering 30144 (2004) 